Editorial

\title{
Special Issue on Current Challenges and Future Directions in Monitoring Responses after Treatment of Primary Cancer
}

\author{
Alexander Stojadinovic ${ }^{1}$, Itzhak Avital 1, Timothy J. Wallace 1, George E. Peoples 2, Scott Steele 2 \\ 1. Bon Secours Cancer Institute, Bon Secours Health System, Richmond, VA, USA \\ 2. Department of Surgery, Uniformed Services University of the Health Sciences, Bethesda, MD, USA
}

\begin{abstract}
$\bowtie$ Corresponding author: stojadinovicmd2011@gmail.com
(c) Ivyspring International Publisher. This is an open-access article distributed under the terms of the Creative Commons License (http://creativecommons.org/ licenses/by-nc-nd/3.0/). Reproduction is permitted for personal, noncommercial use, provided that the article is in whole, unmodified, and properly cited.
\end{abstract}

Published: 2014.01.01

The current special edition of the Journal of Cancer is centered on the present day challenges and future directions in monitoring therapeutic success after completion of primary epithelial cancer therapy. All articles included in this special edition are the work of leading subject matter experts of well-established and reputable institutes in the field of oncology. These articles unequivocally present clinically relevant guidelines; they critically assess commonly encountered challenges in decision making; and, they clearly elucidate effective strategies relevant to monitoring treatment responses of various forms of cancer. In this editorial commentary we recognize and highlight some of these experts' insightful and unique contributions, which may directly benefit our colleagues in clinical practice and research.

The article presented by Dr. Timothy J. Wallace et al. has presented several lines of evidence suggesting that the current practice of ascertaining treatment responses of epithelial malignancies by Response Evaluation Criteria in Solid Tumors (RECIST) is reliable for most solid tumors, with the exception of carcinoma of the prostate (1). There are a number of characteristics about prostate cancer presented by the authors that make the application of RECIST criteria challenging: [1] the size of prostate tumors has been historically difficult to image and measure; [2] when using the RECIST criteria, only $\sim 44 \%$ of men with castrate metastatic and $16 \%$ of non-castrate metastatic prostate cancer have measurable disease; [3] there are no target lesions defined in patients with a rising PSA and localized disease; and, [4] according to RECIST criteria, tumor marker values are recorded as "normal" or "abnormal," whereas monitoring PSA after treatment of prostate cancer occurs across a broad spectrum of PSA values with varying definitions based on which initial treatment modality was administered (1). Based on these facts, the authors have drawn our attention to a number of novel technologies and biomarkers, which appear to be capable of substantially improving our ability to monitor therapeutic response after completion of initial treatment for primary prostate cancer.

The article presented by Dr. William P McGuire's group has concluded that the majority of epithelial ovarian cancer patients will relapse within five years of initial treatment and require salvage systemic therapy (2). Current practice involves long-term surveillance of women for recurrence by clinical exams, serum biomarkers, and imaging. However, there is no evidence that any of these modalities will impact survival as compared to watchful waiting for the presentation of symptoms and initiating treatment at that point in time. However, for many patients the follow-up visit presents an opportunity for medical reassurance, education, managing expectations, and addressing emotional stress and disease-related uncertainty about the likelihood of recurrence despite the inherent limitations of screening tests currently available for epithelial ovarian cancer. Furthermore, patients with a rising serum CA-125 may suffer anxiety, worrying that their dis- 
ease may have recurred, but that treatment is not recommended until symptoms of disease recurrence manifest. The psychological benefit to clinical surveillance is important, and providing these services in a cost-effective manner is paramount. Interventions to proactively reduce patient stress, anxiety and/or depression are certainly an appropriate consideration. The approach to post-treatment surveillance should be individualized taking into account the anticipated clinical benefit of second-line therapy, associated healthcare costs, treatment-related morbidity, and mortality and patient preference.

Articles contributed by Dr. Scott R. Steele's group have reported that the monitoring of colorectal metastases has undergone very few changes in the past decade, though, with improvements in systemic therapy, liver-directed therapy, and with our understanding of how to precisely treat the primary tumor as well as improved treatment approaches to high-risk node-negative patients, overall survival may very well continue to increase $(3,4)$. On the other hand, changes in the way clinicians treat rectal cancer has led to a new age of therapy guided by imaging and pathology. There are indeed paradigm shifts on the horizon for the treatment of rectal cancer. With the ability to predict complete responders before resection of rectal cancer, and compelling data demonstrating comparable long-term, disease-free and overall survival, we may see an evolution toward selected elimination of radical surgery in all except for those who demonstrate local recurrence after neoadjuvant therapy $(3,4)$. This, as well as optimal management of patients presenting with advanced colon cancer, will require precise and accurate modalities to monitor treatment response and individualize selection of treatment.

Articles contributed by George E. Peoples' group have concluded that there are several opportunities to improve how we monitor response to breast cancer treatment $(5,6)$. Only by understanding the current standard of practice regarding all aspects of treatment can we begin to advance the field in a meaningful way. Several fields of cancer care including surgical, radiation and medical oncology, pathology, and radiology work together within integrated practice units to ensure proper study design, selection and coordination of care. They sought to provide a broad overview of these various fields of cancer care, and we believe that future real-world and nimble clinical trials will need to be performed in various clinical pathways discussed in order to provide more granularity and an accelerated pace of discovery for breast cancer researchers $(5,6)$. The current paradigm of first testing new techniques or technologies in the metastatic setting prior to implementation in the adjuvant setting has been challenged by researchers who are committed to accelerating the speed of progress in breast cancer research. Clearly, several opportunities exist to enhance breast cancer care by performing research in the neo-adjuvant setting as outlined in the work presented by Peoples' group $(5,6)$.

Together, these reports clearly indicate that, despite various guidelines currently in use, including RECIST Criteria, MDA Criteria, PET/CT evaluations, NICE guidelines, some of which are well established, not one applies universally across solid organ cancers (1-6). Therefore, personalized and precision medicine-based approaches are warranted. We are in agreement with Dr. Steele et al., such that the "assessment of tumor response is an integral part of this endeavor and plays a pivotal role in the selection of those that will undergo radical surgery and those that should not. While a watch and wait approach cannot currently be encouraged outside of a clinical trial, we must continue to push for novel ideas and techniques with the safety of the patient in mind (3)".

\section{References}

1. Wallace TJ, Torre T, Grob M, Yu J, Avital I, Brücher BLDM, Stojadinovic A, Man YG. Current Approaches, Challenges and Future Directions for Monitoring Treatment Response in Prostate Cancer. J Cancer 2014; 5(1):3-24.

2. Marcus CS, Maxwell GL, Darcy KM, Hamilton CA, McGuire WP. Current Approaches and Challenges in Managing and Monitoring Treatment Response in Ovarian Cancer. J Cancer 2014; 5(1):25-30.

3. McKeown E, Nelson DW, Johnson EK, Maykel JA, Stojadinovic A, Nissan A, Avital I, Brücher BLDM, Steele SR. Current Approaches and Challenges for Monitoring Treatment Response in Colon and Rectal Cancer. J Cancer 2014; 5(1):31-43.

4. Walker AS, Zwintscher NP, Johnson EK, Maykel JA, Stojadinovic A, Nissan A, Avital I, Brücher BLDM, Steele SR. Future Directions for Monitoring Treatment Response in Colorectal Cancer. J Cancer 2014; 5(1):44-57.

5. Graham LJ, Shupe MP, Schneble EJ, Flynt FL, Clemenshaw MN, Kirkpatrick AD, Gallagher C, Nissan A, Henry L, Stojadinovic A, Peoples GE, Shumway NM. Current Approaches and Challenges in Monitoring Treatment Responses in Breast Cancer. J Cancer 2014; 5(1):58-68.

6. Shupe MP, Graham LJ, Schneble EJ, Flynt FL, Clemenshaw MN, Kirkpatrick AD, Stojadinovic A, Peoples GE, Shumway NM. Future Directions for Monitoring Treatment Responses in Breast Cancer. J Cancer 2014; 5(1):69-78. 